\title{
Dynamics of a qubit coupled to a dissipative nonlinear quantum oscillator: An effective-bath approach
}

\author{
Carmen Vierheilig, ${ }^{1}$ Dario Bercioux, ${ }^{2}$ and Milena Grifoni ${ }^{1}$ \\ ${ }^{1}$ Institut für Theoretische Physik, Universität Regensburg, D-93035 Regensburg, Germany \\ ${ }^{2}$ Freiburg Institute for Advanced Studies, Albert-Ludwigs-Universität Freiburg, D-79104 Freiburg im Breisgau, Germany
}

(Received 21 October 2010; published 18 January 2011)

\begin{abstract}
We consider a qubit coupled to a nonlinear quantum oscillator, the latter coupled to an Ohmic bath, and investigate the qubit dynamics. This composed system can be mapped onto that of a qubit coupled to an effective bath. An approximate mapping procedure to determine the spectral density of the effective bath is given. Specifically, within a linear response approximation the effective spectral density is given by knowledge of the linear susceptibility of the nonlinear quantum oscillator. To determine the actual form of the susceptibility, we consider its periodically driven counterpart, which is the problem of the quantum Duffing oscillator within linear response theory in the driving amplitude. Knowing the effective spectral density, the qubit dynamics is investigated. In particular, an analytic formula for the qubit's population difference is derived. Within the regime of validity of our theory, a very good agreement is found with predictions obtained from a Bloch-Redfield master equation approach applied to the composite qubit-nonlinear oscillator system.
\end{abstract}

DOI: 10.1103/PhysRevA.83.012106

PACS number(s): 03.65.Yz, 03.67.Lx, 05.40.-a, 85.25.-j

\section{INTRODUCTION}

The understanding of relaxation and dephasing properties of qubits due to the surrounding environment is essential for quantum computation [1]. A famous model to study the environmental influences on the coherent dynamics of a qubit is the spin-boson model [2-4], consisting of a two-level system (TLS) bilinearly coupled to a bath of harmonic oscillators. Although the bath degrees of freedom can be traced out exactly, analytical solutions are only possible within perturbative schemes. First, those perturbative in the coupling of the TLS to the bath are typically obtained within a Born-Markov treatment of the Liouville equation for the TLS density matrix [5,6] or within the path integral formalism [2]. The equivalence of both methods has been demonstrated under the restriction of low temperatures and low damping strengths in Ref. [7]. The second alternative approach is to perform perturbation theory in the tunneling amplitude of the two-level system. Within the so-called noninteracting blip approximation (NIBA) [2-4] it yields equations of motion for the TLS reduced density matrix that allow it to capture the case of strong TLS-bath coupling. Reality is, however, often more complex, as the qubit might be coupled to other quantum systems besides a thermal bath. For example, to read out its state, a qubit is usually coupled to a read-out device.

In the following we mostly have in mind the flux qubit [8] read out by a dc superconducting quantum interference device (dc SQUID). The latter mediates the dissipation originating from the surrounding electromagnetic bath and can be modeled both as a linear or nonlinear oscillator [8-19]. Recently, the nonlinearity of qubit read-out devices, for example of a dc SQUID [15-19] or a Josephson bifurcation amplifier [20-22], has been used to improve the measurement scheme in terms of a faster read out and higher fidelity. Specifically, the device was operated in a regime where the dynamics exhibited bifurcation features typical of a classical nonlinear oscillator. As demonstrated in, for example, Ref. [12], the quantum limit is within experimental reach as well.
From the theoretical side, there are two different viewpoints to investigate the dynamics of a qubit coupled to an oscillator, with the latter in turn coupled to a thermal bath. The first way is to consider the TLS and the oscillator as a single quantum system coupled to the bath, while the second is an effectivebath description where the effective environment seen by the qubit includes the oscillator and the original thermal bath. The mapping to an effective bath has been discussed for the case in which the TLS is coupled to a harmonic oscillator in Ref. [23]. Specifically, the spectral density of the effective bath acquires a broadened peak centered around the frequency of the oscillator. This case has been investigated in Refs. [24-30] by applying standard numerical and analytical methods established for the spin-boson model. All those works showed that the peaked structure of the effective bath is essential when the eigenfrequency of the TLS becomes comparable to the oscillator frequency.

So far, the first approach was used in Ref. [31] to describe a qubit-nonlinear oscillator (NLO) system in the deep quantum regime. Here the effects of the (harmonic) thermal reservoir can be treated using standard Born-Markov perturbation theory. The price to be paid, however, is that the Hilbert space of the qubit-nonlinear oscillator system is infinite, which requires, for practical calculations, its truncation, invoking, for example, low temperatures [31].

In contrast to the above work, we investigate here the case of a qubit-NLO system, with the latter being coupled to an Ohmic bath, within an effective-bath description. Due to the nonlinearity of the oscillator, the mapping to a linear effective bath is not exact. In this case a temperature and nonlinearitydependent effective spectral density well captures the NLO influence on the qubit dynamics.

This article is organized as follows: In Sec. II we introduce the model with the relevant quantities. In Sec. III the mapping procedure is investigated and the effective spectral density for the corresponding linear case is given. Afterwards, the mapping procedure is applied to the case of a qubit coupled to a nonlinear quantum oscillator. As a consequence of the 
mapping, the determination of the effective spectral density is directly related to the knowledge of the susceptibility of the oscillator. We show how the susceptibility can be obtained from the steady-state response of a quantum Duffing oscillator in Sec. III C. In Sec. IV the steady-state response of the dissipative quantum Duffing oscillator is reviewed and its susceptibility is put forward. The related effective spectral density is derived in Sec. V. In Sec. VI the qubit dynamics is investigated by applying the noninteracting blip approximation (NIBA) to the kernels of the generalized master equation which governs the dynamics of the population difference of the qubit. A comparison with the results of Ref. [31], obtained within the first approach, is shown. Furthermore, analogies and differences with respect to the linear case are discussed. In Sec. VII, conclusions are drawn.

\section{HAMILTONIAN}

We consider a composed system built of a qubit-the system of interest - coupled to a nonlinear quantum oscillator (NLO); see Fig. 1. To read-out the qubit state we couple the qubit linearly to the oscillator with the coupling constant $\bar{g}$ such that, via the intermediate NLO, dissipation also enters the qubit dynamics. The Hamiltonian of the composed system reads

$$
\hat{H}_{\mathrm{tot}}=\hat{H}_{\mathrm{S}}+\hat{H}_{\mathrm{NLO}}+\hat{H}_{\mathrm{S}+\mathrm{NLO}}+\hat{H}_{\mathrm{NLO}+\mathrm{B}}+\hat{H}_{\mathrm{B}},
$$

where

$$
\begin{gathered}
\hat{H}_{\mathrm{S}}=\frac{\hat{p}^{2}}{2 \mu}+U(\hat{q}), \\
\hat{H}_{\mathrm{NLO}}=\frac{1}{2 M} \hat{P}_{y}^{2}+\frac{1}{2} M \Omega^{2} \hat{y}^{2}+\frac{\bar{\alpha}}{4} \hat{y}^{4}, \\
\hat{H}_{\mathrm{S}+\mathrm{NLO}}=\bar{g} \hat{y} \hat{q}, \\
\hat{H}_{\mathrm{NLO}+\mathrm{B}}=\sum_{j}\left[-c_{j} \hat{x}_{j} \hat{y}+\frac{c_{j}^{2}}{2 m_{j} \omega_{j}^{2}} \hat{y}^{2}\right], \\
\hat{H}_{\mathrm{B}}=\sum_{j}\left[\frac{\hat{p}_{j}^{2}}{2 m_{j}}+\frac{1}{2} m_{j} \omega_{j}^{2} \hat{x}_{j}^{2}\right] .
\end{gathered}
$$

Here, $\hat{H}_{\mathrm{S}}$ represents the qubit Hamiltonian, where $\mu$ is the particle's mass and $U(q)$ a one-dimensional double well potential with minima at $q= \pm q_{0} / 2 . \hat{H}_{\mathrm{NLO}}$ is the NLO Hamiltonian, where the parameter $\bar{\alpha}>0$ accounts for the nonlinearity. When the oscillator represents a SQUID used to read out the qubit, the oscillator frequency $\Omega$ corresponds to the SQUID's plasma frequency. The dissipation in the NLO is modeled in the following by coupling it to an Ohmic bath

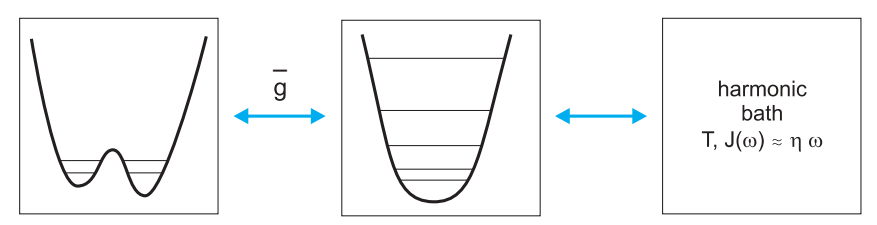

FIG. 1. (Color online) Schematic representation of the composed system built of a qubit, an intermediate nonlinear oscillator, and an Ohmic bath.

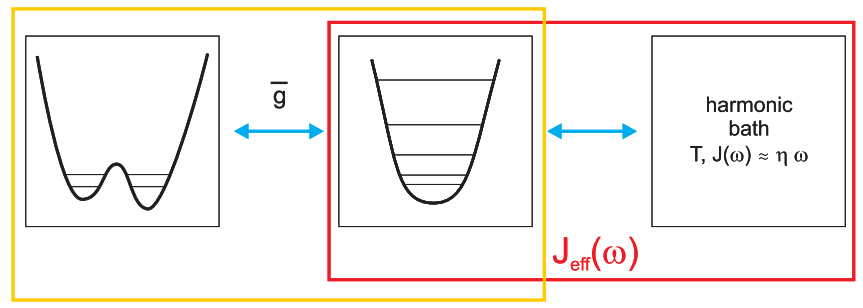

FIG. 2. (Color online) Schematic representation of the complementary approaches available to evaluate the qubit dynamics. In the first approach one determines the eigenvalues and eigenfunctions of the composite qubit plus oscillator system [yellow (light gray) box] and accounts afterwards for the harmonic bath characterized by the Ohmic spectral density $J(\omega)$. In the effective-bath description, one considers an environment built of the harmonic bath and the nonlinear oscillator [red (dark gray) box]. In the harmonic approximation, the effective bath is fully characterized by its effective spectral density $J_{\text {eff }}(\omega)$.

characterized by the spectral density [2]

$$
J(\omega)=\frac{\pi}{2} \sum_{j=1}^{\mathcal{N}} \frac{c_{j}^{2}}{m_{j} \omega_{j}} \delta\left(\omega-\omega_{j}\right)=\eta \omega=M \gamma \omega .
$$

In the classical limit it corresponds to a white noise source, where $\eta$ is a friction coefficient with dimensions of mass times frequency.

In the following, focus will be on the qubit dynamics in the presence of the dissipative nonlinear oscillator. Namely, we will study the time evolution of the qubit's position as described by

$$
q(t):=\operatorname{Tr}\left\{\hat{\rho}_{\text {tot }}(t) \hat{q}\right\}=\operatorname{Tr}_{\mathrm{S}}\left\{\hat{\rho}_{\text {red }}(t) \hat{q}\right\}
$$

where $\hat{\rho}_{\text {tot }}$ and $\hat{\rho}_{\text {red }}$ are the total and reduced density operators, respectively. The latter is defined as

$$
\hat{\rho}_{\text {red }}:=\operatorname{Tr}_{\mathrm{B}} \operatorname{Tr}_{\mathrm{NLO}}\left\{\hat{\rho}_{\mathrm{tot}}\right\}
$$

where the trace over the degrees of freedom of the bath and of the oscillator is taken. In Fig. 2, two different approaches to determine the qubit dynamics are depicted. In the first approach, which is elaborated in Ref. [31], one first determines the eigenstates and eigenvalues of the composed qubitoscillator system and then includes environmental effects via standard Born-Markov perturbation theory. The second approach exploits an effective description for the environment surrounding the qubit and is based on a mapping procedure. This will be investigated in the next section.

\section{MAPPING}

The main aim is to evaluate the qubit's evolution described by $q(t)$. This can be achieved within an effective description using a mapping procedure. Thereby, the oscillator and the Ohmic bath are put together, as depicted in Fig. 2, to form an effective bath. The effective Hamiltonian

$$
\hat{H}_{\text {eff }}=\hat{H}_{\mathrm{S}}+\hat{H}_{\mathrm{B} \text { eff }}
$$

is chosen such that, after tracing out the bath degrees of freedom, the same dynamical equations for $q(t)$ are obtained as from the original Hamiltonian $\hat{H}_{\text {tot }}$. Due to the nonlinear 
character of the oscillator, an exact mapping implies that $\hat{H}_{\mathrm{B} \text { eff }}$ represents a nonlinear environment. We shall show in the following subsection using linear response theory that a linear approximation for $\hat{H}_{\mathrm{B} \text { eff }}$ is justified for weak coupling $\bar{g}$. Then Eq. (6) describes an effective spin-boson problem where

$$
\hat{H}_{\mathrm{B} \text { eff }}=\frac{1}{2} \sum_{j=1}^{\mathcal{N}}\left[\frac{\hat{P}_{j}^{2}}{m_{j}}+m_{j} \omega_{j}^{2}\left(\hat{X}_{j}-\frac{d_{j}}{m_{j} \omega_{j}^{2}} \hat{q}\right)^{2}\right],
$$

and the associated spectral density is

$$
J_{\text {eff }}(\omega)=\frac{\pi}{2} \sum_{j=1}^{\mathcal{N}} \frac{d_{j}^{2}}{m_{j} \omega_{j}} \delta\left(\omega-\omega_{j}\right) .
$$

The Hamiltonian (6) with (7) leads to coupled equations of motion [2,3]:

$$
\begin{gathered}
\mu \ddot{\hat{q}}(t)+U^{\prime}(\hat{q})+\sum_{j=1}^{\mathcal{N}}\left(\frac{d_{j}^{2}}{m_{j} \omega_{j}^{2}} \hat{q}\right)=\sum_{j=1}^{\mathcal{N}} d_{j} \hat{X}_{j}, \\
m_{j} \ddot{\hat{X}}_{j}+m_{j} \omega_{j}^{2} \hat{X}_{j}=d_{j} \hat{q},
\end{gathered}
$$

where $U^{\prime}(\hat{q})=\frac{d}{d q} U(\hat{q})$. By formally integrating the second equation of motion and inserting the solution into the first equation, the well-known Langevin equation for the operator $\hat{q}$ is obtained. This, in turn, leads to the Langevin equation for $q_{\mathrm{eff}}(t):=\operatorname{Tr}\left\{\hat{\rho}_{\mathrm{eff}} \hat{q}(t)\right\}[2]:$

$$
\mu \ddot{q}_{\mathrm{eff}}+\mu \int_{0}^{t} d t^{\prime} \gamma_{\mathrm{eff}}\left(t-t^{\prime}\right) \dot{q}_{\mathrm{eff}}+\left\langle U^{\prime}(\hat{q})\right\rangle_{\mathrm{eff}}=0,
$$

with the effective damping kernel $\gamma_{\mathrm{eff}}\left(t-t^{\prime}\right)$.

Notice that $\langle\ldots\rangle_{\mathrm{eff}}$ indicates the expectation value taken with respect to $\hat{\rho}_{\text {eff }}$, which is the density operator associated to $\hat{H}_{\text {eff }}$ [2]. In Laplace space, defined by

$$
\begin{gathered}
y(t)=\frac{1}{2 \pi i} \int_{\mathcal{C}} d \lambda y(\lambda) \exp (\lambda t), \\
y(\lambda)=\int_{0}^{\infty} d t y(t) \exp (-\lambda t),
\end{gathered}
$$

we obtain from Eq. (9) the equation of motion

$$
\mu \lambda^{2} q_{\mathrm{eff}}(\lambda)+\mu \lambda \gamma_{\mathrm{eff}}(\lambda) q_{\mathrm{eff}}(\lambda)+\left\langle U^{\prime}(\lambda)\right\rangle_{\mathrm{eff}}=0 .
$$

The real part $\gamma_{\text {eff }}^{\prime}(\omega)=\operatorname{Re}\left[\gamma_{\text {eff }}(\lambda=-i \omega)\right]$ of the effective damping kernel $\gamma_{\mathrm{eff}}(t)$ is related to the spectral density via [2]

$$
\gamma_{\mathrm{eff}}^{\prime}(\omega)=\frac{J_{\mathrm{eff}}(\omega)}{\mu \omega}
$$

The mapping for the case of zero nonlinearity $\bar{\alpha}$ and Ohmic damping has been discussed in Ref. [23]. There, the influence of both the intermediate harmonic oscillator and the bath is embedded into an effective peaked spectral density given by

$$
J_{\mathrm{eff}}^{\mathrm{HO}}(\omega)=\frac{\bar{g}^{2} \gamma \omega}{M\left(\Omega^{2}-\omega^{2}\right)^{2}+M \gamma^{2} \omega^{2}},
$$

showing Ohmic low-frequency behavior $J_{\text {eff }}^{\mathrm{HO}}(\omega) \underset{\omega \rightarrow 0}{\longrightarrow}$ $\bar{g}^{2} \gamma \omega /\left(M \Omega^{4}\right)$.

\section{A. Equation of motion for the nonlinear Hamiltonian}

As discussed above, the mapping requires knowledge of the reduced dynamics of the system described by the variable $q(t)$. Therefore, we start from the coupled equations of motion derived from the Hamiltonian $\hat{H}_{\text {tot }}$ given in Eq. (1):

$$
\begin{gathered}
\mu \ddot{\hat{q}}+U^{\prime}(\hat{q})=-\bar{g} \hat{y}, \\
M \ddot{\hat{y}}+\eta \dot{\hat{y}}+M \Omega^{2} \hat{y}+\bar{\alpha} \hat{y}^{3}=-\bar{g} \hat{q}+\hat{\xi}(t) .
\end{gathered}
$$

According to Eq. (3), $\eta=M \gamma$ is the damping coefficient and

$\hat{\xi}(t)=\sum_{j=1}^{\mathcal{N}} c_{j}\left[x_{j}^{(0)} \cos \left(\omega_{j} t\right)+\frac{p_{j}^{(0)}}{m_{j} \omega_{j}} \sin \left(\omega_{j} t\right)\right]-M \gamma \delta(t) \hat{y}(0)$

is a fluctuating force originating from coupling to the bath. In order to eliminate $\hat{y}$ from the first equation of motion, we have to calculate $\hat{y}[\hat{q}(t)]$ from the second equation.

In the following, we look at equations of motion for the expectation values resulting from Eqs. (14a) and (14b); that is, we look at the evolution of $q(t):=\operatorname{Tr}\left\{\hat{\rho}_{\text {tot }} \hat{q}(t)\right\}$ and $y(t):=$ $\operatorname{Tr}\left\{\hat{\rho}_{\text {tot }} \hat{y}(t)\right\}$. Since we want to calculate $y(t)$, we turn back to Eq. (1) and treat the coupling term $\hat{H}_{\mathrm{S}+\mathrm{NLO}}$ as a perturbation, $\bar{g} y_{0} q_{0} \ll \hbar \Omega$, where we introduced the oscillator length $y_{0}=$ $\sqrt{\hbar /(M \Omega)}$. Then, the use of linear response theory in this perturbation is justified, and we find

$$
\begin{aligned}
y(t)= & \langle\hat{y}(t)\rangle_{0}-\frac{i}{\hbar} \int_{-\infty}^{\infty} d t^{\prime} \theta\left(t-t^{\prime}\right)\left\langle\left[\hat{y}(t), \hat{y}\left(t^{\prime}\right)\right]\right\rangle_{0} \bar{g}\left\langle\hat{q}\left(t^{\prime}\right)\right\rangle_{0} \theta\left(t^{\prime}\right) \\
& +O\left(\bar{\alpha} \bar{g}^{2}\right),
\end{aligned}
$$

where $\langle\ldots\rangle_{0}$ denotes the expectation value in the absence of the coupling $\bar{g}$, which we assume has been switched on at time $t_{0}=0$.

Notice that, for a linear system (for example, the damped harmonic oscillator), the linear response becomes exact, such that the neglected corrections are at least of order $O\left(\bar{\alpha} \bar{g}^{2}\right)$. Moreover, the time evolution of the expectation values is the same as in the classical case; this fact corresponds to the Ehrenfest theorem [2]. For nonlinear systems, the expression in Eq. (16) is an approximation because all orders in the perturbation are nonvanishing. ${ }^{1}$

In Laplace space, Eq. (16) yields

$$
\delta y(\lambda)=\chi(\lambda) \bar{g}\langle\hat{q}(\lambda)\rangle_{0}+O\left(\bar{\alpha} \bar{g}^{2}\right),
$$

where $\delta y(\lambda)=y(\lambda)-\langle\hat{y}(\lambda)\rangle_{0}$ and where $\chi(\lambda)$ is the Laplace transform of the response function or susceptibility

$$
\chi\left(t-t^{\prime}\right)=-\frac{i}{\hbar} \theta\left(t-t^{\prime}\right)\left\langle\left[\hat{y}(t), \hat{y}\left(t^{\prime}\right)\right]\right\rangle_{0} .
$$

Since $q(\lambda)-\langle\hat{q}(\lambda)\rangle_{0}=O(\bar{g})$, from Eqs. (14a) and (17) it follows that

$$
\begin{aligned}
& \mu \lambda^{2} q(\lambda)+\bar{g}^{2} \chi(\lambda) q(\lambda)+O\left(\bar{\alpha} \bar{g}^{3}, \bar{g}^{3}\right) \\
& \quad=-\left\langle U^{\prime}(\lambda)\right\rangle-\bar{g}\langle\hat{y}(\lambda)\rangle_{0} .
\end{aligned}
$$

\footnotetext{
${ }^{1}$ An extension of the concept of linear response in case of nonlinear systems is the so-called Volterra expansion, which provides a systematic perturbation series in the forcing [32].
} 
That is, we have a normalization of the mass, and a dampinglike term due to the coupled equations of motion. The effect of the nonlinearity is embedded in the response function $\chi$.

We assume in the following that in the absence of the coupling to the qubit the NLO and bath are in thermal equilibrium, which yields $\langle\hat{y}(t)\rangle_{0}=0$ for all times and, thus, also $\langle\hat{y}(\lambda)\rangle_{0}=0$.

\section{B. Mapping of the equations of motion and generic form for the effective spectral density}

By comparison of Eqs. (11) and (19) we can conclude that they yield the same dynamics if

$$
\left\langle U^{\prime}(\lambda)\right\rangle_{\mathrm{eff}}=\left\langle U^{\prime}(\lambda)\right\rangle,
$$

and the effective bath is chosen such that

$$
\bar{g}^{2} \frac{\chi(\lambda)}{\mu \lambda}=\gamma_{\mathrm{eff}}(\lambda) .
$$

By comparing the last equations with the relationship (12) and replacing $\lambda=-i \omega$, it follows that

$$
J_{\text {eff }}(\omega)=-\bar{g}^{2} \chi^{\prime \prime}(\omega),
$$

where $\chi^{\prime \prime}(\omega)$ is the imaginary part of the susceptibility in Fourier space. We have now reduced the problem of finding the effective spectral density to that of determining the corresponding susceptibility. Notice that, for a linear system, the classical and quantum susceptibility coincide and are independent of the driving amplitude! In this case it is possible to calculate $\chi(\omega)$ directly from the classical equations of motion. For a generic nonlinear system, however, the classical and quantum susceptibilities differ.

\section{Linear susceptibility of a Duffing oscillator}

In order to evaluate the linear susceptibility, we solve the auxiliary problem of calculating the susceptibility of a quantum Duffing oscillator (DO); that is, of the nonlinear quantum oscillator in Eq. (2) additionally driven by a periodic force with driving amplitude $F$ and driving frequency $\omega_{\mathrm{ex}}$. The corresponding equation of motion is

$$
M \ddot{\hat{y}}+\eta \dot{\hat{y}}+M \Omega^{2} \hat{y}+\bar{\alpha} \hat{y}^{3}=-F \theta\left(t-t_{0}\right) \cos \left(\omega_{\mathrm{ex}} t\right)+\hat{\xi}(t) .
$$

Application of linear response theory in the driving yields the equation for the expectation value of the position of the oscillator:

$$
\begin{aligned}
y(t)= & \langle\hat{y}(t)\rangle_{0}-\frac{i}{\hbar} \int_{t_{0}}^{\infty} d t^{\prime} \theta\left(t-t^{\prime}\right)\left\langle\left[\hat{y}(t), \hat{y}\left(t^{\prime}\right)\right]\right\rangle_{0} F \cos \left(\omega_{\mathrm{ex}} t^{\prime}\right) \\
& +O\left(F^{2}\right) .
\end{aligned}
$$

Using the symmetry properties of the susceptibility $\chi(\omega)$, we obtain in the steady-state limit

$$
\begin{aligned}
y_{\mathrm{st}}(t)= & \lim _{t_{0} \rightarrow-\infty} y(t)=\langle\hat{y}(t)\rangle_{0}+F \cos \left(\omega_{\mathrm{ex}} t\right) \chi^{\prime}\left(\omega_{\mathrm{ex}}\right) \\
& +F \sin \left(\omega_{\mathrm{ex}} t\right) \chi^{\prime \prime}\left(\omega_{\mathrm{ex}}\right)+O\left(F^{3}\right) \\
\equiv & A \cos \left(\omega_{\mathrm{ex}} t+\phi\right)+O\left(F^{3}\right)
\end{aligned}
$$

Here, the presence of the Ohmic bath implies $\lim _{t_{0} \rightarrow-\infty}$ $\langle\hat{y}(t)\rangle_{0}=0$. Notice that, due to symmetry inversion of the NLO, corrections of $O\left(F^{2}\right)$ vanish in Eq. (25). In Eq. (25), $A$ and $\phi$ are the amplitude and phase of the steady-state response, respectively. It follows that $\chi(\omega)=\frac{A}{F} \exp (-i \phi)$, such that $\chi^{\prime \prime}(\omega)=-\frac{A}{F} \sin \phi$.

\section{STEADY-STATE DYNAMICS OF A DUFFING OSCILLATOR}

So far we have reduced the problem of finding the effective spectral density to the one of determining the steady-state response of the Duffing oscillator in terms of the amplitude $A$ and the phase $\phi$. These quantities were recently derived in Refs. [33,34], using the framework of a Bloch-Redfield-Floquet description of the dynamics of the DO. The results in Ref. [34] are applicable in a wide range of driving frequencies around the one-photon resonance regime $\omega_{\mathrm{ex}}=\Omega+3 \bar{\alpha} y_{0}^{4} /(4 \hbar) \equiv \Omega_{1}$ for sufficiently strong nonlinearities: $y_{0} F /(2 \sqrt{2}) \ll 3 \bar{\alpha} y_{0}^{4} / 4 \ll \hbar \Omega$.

As illustrated in Refs. [33,34], the amplitude and phase are fully determined by knowledge of the matrix elements of the stationary density matrix of the Duffing oscillator in the Floquet basis [see, e.g., Eqs. (67)-(70) in Ref. [34]]. There, the master equation yielding the elements of the stationary density matrix is analytically solved in the low-temperature regime $k_{B} T \ll \hbar \Omega$ imposing a partial secular approximation, yielding Eq. (70) of Ref. [34], and restricting to spontaneous emission processes only. Here, we follow the same line of reasoning as in Ref. [34] to evaluate the amplitude and phase: we impose the same partial secular approximation and consider low temperatures $k_{B} T<\hbar \Omega$. However, we include now both emission and absorption processes; that is, we use the full dissipative transition rates as in Eq. (64) of Ref. [34]. The imaginary part of the linear susceptibility $\chi$ follows from the thus-obtained nonlinear susceptibility $\chi_{N L}$ in the limit of vanishing driving amplitudes:

$$
\begin{aligned}
& \chi^{\prime \prime}\left(\omega_{\mathrm{ex}}\right) \\
& =\lim _{F \rightarrow 0} \chi_{N L}^{\prime \prime}\left(\omega_{\mathrm{ex}}\right) \\
& =-\frac{y_{0}^{4} J\left(\omega_{\mathrm{ex}}\right) n_{1}(0)^{4} \frac{2 \Omega_{1}}{\left|\omega_{\mathrm{ex}}\right|+\Omega_{1}}}{y_{0}^{4} J\left(\Omega_{1}\right)^{2} n_{1}(0)^{4}\left[2 n_{\mathrm{th}}\left(\Omega_{1}\right)+1\right]^{2}+4 \hbar^{2}\left(\left|\omega_{\mathrm{ex}}\right|-\Omega_{1}\right)^{2}},
\end{aligned}
$$

where

$$
n_{1}(0)=\left[1-\frac{3}{8 \hbar \Omega} \bar{\alpha} y_{0}^{4}\right] .
$$

For consistency, $n_{1}^{4}(0)$ also has to be treated up to first order in $\bar{\alpha}$ only.

Moreover, we used the spectral density $J(\omega)=M \gamma \omega$ and the Bose function $n_{\mathrm{th}}(\epsilon)=\left\{\operatorname{coth}\left[\hbar \epsilon /\left(2 k_{B} T\right)\right]-1\right\} / 2$, which determines the weight of the emission and absorption processes.

\section{EFFECTIVE SPECTRAL DENSITY FOR A NONLINEAR SYSTEM}

The effective spectral density follows from Eqs. (22) and (26). It reads

$$
\begin{aligned}
& J_{\text {eff }}\left(\omega_{\mathrm{ex}}\right) \\
& =\bar{g}^{2} \frac{\gamma \omega_{\mathrm{ex}} n_{1}(0)^{4} \frac{2 \Omega_{1}}{\left|\omega_{\mathrm{ex}}\right|+\Omega_{1}}}{M \gamma^{2} \Omega_{1}^{2}\left[2 n_{\mathrm{th}}\left(\Omega_{1}\right)+1\right]^{2} n_{1}(0)^{4}+4 M \Omega^{2}\left(\left|\omega_{\mathrm{ex}}\right|-\Omega_{1}\right)^{2}} .
\end{aligned}
$$




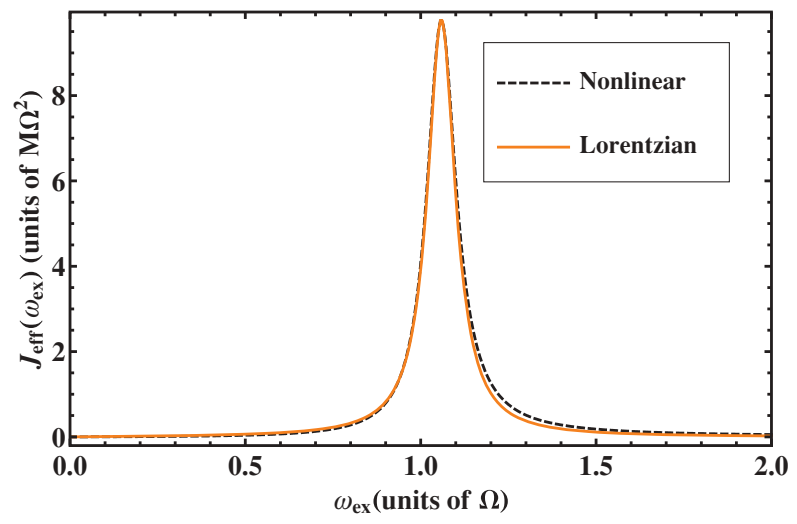

FIG. 3. (Color online) Comparison of the effective spectral density $J_{\text {eff }}(\omega)$ and a Lorentz curve for moderate damping. The parameters are $\Omega=1.0, y_{0}^{4} \bar{\alpha} /(\hbar \Omega)=0.08, \gamma=0.097 \Omega$, and $\beta=$ $10(\hbar \Omega)^{-1}$.

As in the case of the effective spectral density $J_{\text {eff }}^{\mathrm{HO}}$ [Eq. (13)], we observe Ohmic behavior at low frequency. In contrast to the linear case, the effective spectral density is peaked at the shifted frequency $\Omega_{1}$. Its shape approaches the Lorentzian one of the linear effective spectral density, but with a peak at the shifted frequency, as shown in Fig. 3. While in Refs. [33,34], the amplitude of the oscillator showed an antiresonant-to-resonant transition depending on the ratio of driving amplitude $F$ and damping $\gamma$ the effective spectral density, obtained in the limit $F \rightarrow 0$, displays only resonant behavior.

\section{QUBIT DYNAMICS}

In the following we derive the dynamics of a qubit coupled to this effective nonlinear bath. Therefore, we identify the system Hamiltonian $\hat{H}_{\mathrm{S}}$ introduced in Eq. (1) with the one of a qubit, denoted in the following as $\hat{H}_{\mathrm{TLS}}$. This is verified at low energies if the barrier height of the double well potential $U(\hat{q})$ is larger than the energy separation of the ground and first excited levels in each well. In this case, the relevant Hilbert space can be restricted to the two-dimensional space spanned by the ground-state vectors $|L\rangle$ and $|R\rangle$ in the left and right potential well, respectively [2]. We start by defining the actual form of the qubit Hamiltonian and its interaction with the nonlinear oscillator and afterwards introduce its dynamical quantity of interest, which is the population difference $P(t)$.

\section{A. Qubit}

The Hamiltonian of the TLS (qubit), given in the localized basis $\{|L\rangle,|R\rangle\}$, is

$$
\hat{H}_{\mathrm{TLS}}=-\frac{\hbar}{2}\left(\varepsilon \sigma_{z}+\Delta \sigma_{x}\right)
$$

where $\sigma_{i}, i=x, z$, are the corresponding Pauli matrices, the energy bias $\varepsilon$ accounts for an asymmetry between the two wells, and $\Delta$ is the tunneling amplitude. The bias $\varepsilon$ can be tuned for a superconducting flux qubit by application of an external flux $\Phi_{\text {ext }}$ and vanishes at the so-called degeneracy point [35]. For $\varepsilon \gg \Delta$ the states $|L\rangle$ and $|R\rangle$ are eigenstates of $\hat{H}_{\mathrm{TLS}}$, corresponding to clockwise and counterclockwise currents, respectively.

The interaction in Eq. (2) is conveniently rewritten as

$$
\hat{H}_{\mathrm{TLS}-\mathrm{NLO}}=\bar{g} \hat{q} \hat{y}:=\hbar g \sigma_{z}\left(a+a^{\dagger}\right),
$$

with $\hbar g=\frac{\bar{g}}{2 \sqrt{2}} q_{0} y_{0}$. Likewise, we express the nonlinearoscillator Hamiltonian as

$$
\begin{aligned}
\hat{H}_{\mathrm{NLO}} & =\hbar \Omega\left(\hat{j}+\frac{1}{2}\right)+\frac{\bar{\alpha}}{4} \hat{y}^{4} \\
& :=\hbar \Omega\left(\hat{j}+\frac{1}{2}\right)+\frac{\alpha}{4}\left(a+a^{\dagger}\right)^{4},
\end{aligned}
$$

where $\alpha=\bar{\alpha} y_{0}^{4} / 4$.

\section{B. Population difference}

The dynamics of a qubit is usually characterized in terms of the population difference $P(t)$ between the $|R\rangle$ and $|L\rangle$ states of the qubit:

$$
P(t):=\left\langle\sigma_{z}\right\rangle=\operatorname{Tr}_{\mathrm{TLS}}\left\{\sigma_{\mathrm{z}} \hat{\rho}_{\mathrm{red}}(t)\right\},
$$

where $\hat{\rho}_{\text {red }}(t)$ is the reduced density matrix of the TLS:

$$
\hat{\rho}_{\text {red }}(t)=\operatorname{Tr}_{\mathrm{B}}\left\{\hat{\rho}_{\text {eff }}(t)\right\} .
$$

It is found after tracing out the degrees of freedom of the effective bath from the total density matrix, $\hat{\rho}_{\text {eff }}(t)=$ $\exp ^{-\frac{i}{\hbar} \hat{H}_{\text {eff }} t} \hat{\rho}_{\text {eff }}(0) \exp ^{\frac{i}{\hbar}} \hat{H}_{\text {eff }} t$. It follows that, in the two-level approximation, $q_{\mathrm{eff}}(t)=\frac{q_{0}}{2} P(t)$, where $q_{\mathrm{eff}}(t)$ is the position operator expectation value introduced in Sec. III.

As we mapped the nonlinear system onto an effective spin-boson model, the evaluation of the population difference $P(t)$ of the TLS is possible using standard approximations developed for the spin-boson model [25,26,28]. Assuming a factorized initial condition $\hat{\rho}_{\mathrm{eff}}(0)=\hat{\rho}_{\mathrm{TLS}}(0) Z^{-1} \exp \left(-\beta \hat{H}_{\mathrm{B} \text { eff }}\right)$, the population difference $P(t)$ fulfills the generalized master equation (GME) [2,36]

$$
\dot{P}(t)=-\int_{0}^{t} d t^{\prime}\left[K^{s}\left(t-t^{\prime}\right) P\left(t^{\prime}\right)+K^{a}\left(t^{\prime}\right)\right], \quad t>0,
$$

where $K^{s}\left(t-t^{\prime}\right)$ and $K^{a}\left(t-t^{\prime}\right)$ are symmetric and antisymmetric with respect to the bias, respectively. They are represented as a series in the tunneling amplitude. Because neither an analytic nor a numeric solution is available due to the complicated form of the exact kernel, we impose in the following the so-called noninteracting blip approximation (NIBA) [2,3]. Applying the NIBA corresponds to truncating the exact kernels to first order in $\Delta^{2}$ and is therefore perturbative in the tunneling amplitude of the qubit. It is justified in various regimes: It is exact at zero damping, otherwise it is only an approximation which works at best for zero bias and/or large damping and/or high temperature [2]. One finds within the NIBA:

$$
\begin{aligned}
K^{s}(t) & =\Delta^{2} \exp [-S(t)] \cos [R(t)], \\
K^{a}(t) & =\Delta^{2} \exp [-S(t)] \sin [R(t)],
\end{aligned}
$$


where $S(\tau)$ and $R(\tau)$ are, respectively, the real and imaginary part of the bath correlation function:

$$
\begin{aligned}
Q(\tau)= & S(\tau)+i R(\tau)=\int_{0}^{\infty} d \omega \frac{G_{\mathrm{eff}}(\omega)}{\omega^{2}} \\
& \times\left[\operatorname{coth}\left\{\frac{\beta \hbar \omega}{2}[1-\cos (\omega t)]\right\}+i \sin (\omega t)\right],
\end{aligned}
$$

where $G_{\text {eff }}(\omega)=q_{0}^{2} J_{\text {eff }}(\omega) /(\pi \hbar)$. In particular, upon introducing the dimensionless constant $\varsigma=g^{2} \gamma n_{1}(0)^{4} /\left(\pi \Omega^{3}\right)$, we obtain

$$
G_{\mathrm{eff}}(\omega)=2 \varsigma \Omega^{2} \frac{\omega \frac{2 \Omega_{1}}{|\omega|+\Omega_{1}}}{\bar{\gamma}^{2}+\left(|\omega|-\Omega_{1}\right)^{2}},
$$

where we used $\Omega_{1} n_{1}(0)^{2}=\Omega+O\left(\alpha^{2}\right)$ and $\bar{\gamma}_{\text {th }}:=$ $\left[2 n_{\text {th }}\left(\Omega_{1}\right)+1\right] \gamma / 2$. Consequently, the dynamics of the qubit is fully determined by the knowledge of the correlation function $Q(\tau)$ and hence of the effective spectral density derived in Sec. V. The explicit form of $Q(\tau)$ for the case of the effective nonlinear bath is given in Appendix.

\section{Analytical solution for the nonlinear peaked spectral density}

In this section we derive an analytical formula for the population difference $P(t)$ for the symmetric case $(\varepsilon=0)$, requiring weak damping strengths $\gamma$, such that a weak damping approximation of the NIBA kernels is verified; specifically, $\gamma /(2 \pi \Omega) \ll 1$. As this calculation is analogous to the one illustrated in detail in Ref. [28], we only define the relevant quantities and give the main results.

Due to the convolutive form of Eq. (34), this integrodifferential equation is solved by applying a Laplace transform. In Laplace space it reads

$$
P(\lambda)=\frac{1-\frac{1}{\lambda} K^{a}(\lambda)}{\lambda+K^{s}(\lambda)},
$$

where $P(\lambda)=\int_{0}^{\infty} d t \exp (-\lambda t) P(t)$ and analogously for $K^{a / s}(\lambda)$.

Consequently, the dynamics of $P(t)$ is determined if the poles of

$$
\lambda+K^{s}(\lambda)=0
$$

are found and the corresponding back-transformation is applied. We transform the kernels in Eq. (35) in Laplace space and expand them up to first order in the damping. This procedure is called the weak damping approximation (WDA) in Ref. [28]. One obtains

$$
\begin{gathered}
K^{(s)}(\lambda)=\Delta^{2} \int_{0}^{\infty} d \tau \exp (-\lambda \tau) \exp \left[-S_{0}(\tau)\right] \\
\times\left\{\cos \left[R_{0}(\tau)\right]\left[1-S_{1}(\tau)\right]-\sin \left[R_{0}(\tau)\right] R_{1}(\tau)\right\} \\
K^{(a)}(\lambda)=0,
\end{gathered}
$$

where the indices $\{0,1\}$ denote the actual order in the damping. Specifically,

$$
\begin{aligned}
S(\tau) & =S_{0}(\tau)+S_{1}(\tau)+O\left(\gamma^{2}\right), \\
R(\tau) & =R_{0}(\tau)+R_{1}(\tau)+O\left(\gamma^{2}\right),
\end{aligned}
$$

where the explicit expression of the above correlation functions is found in Appendix.

With this we are able to solve the pole equation for $P(t)$ [Eq. (39)] as an expansion up to first order in the damping around the solutions $\lambda_{p}$ of the noninteracting pole equation; that is, $\lambda^{*}=\lambda_{p}-\gamma \kappa_{p}+i \gamma v+O\left(\gamma^{2}\right)$, as $\gamma / \Omega \ll 1$. Following Nesi et al. [28], the kernel is rewritten in the compact form

$$
\begin{aligned}
K^{(s)}(\lambda)= & \sum_{n=0}^{\infty} \int_{0}^{\infty} d \tau \exp (-\lambda \tau)\left\{\Delta_{n, c}^{2} \cos \left(n \Omega_{1} t\right)\left[1-S_{1}(\tau)\right]\right. \\
& \left.+\Delta_{n, s}^{2} \sin \left(n \Omega_{1} t\right) R_{1}(\tau)\right\}
\end{aligned}
$$

where

$$
\Delta_{n, c}=\Delta \exp (Y / 2) \sqrt{\left(2-\delta_{n, 0}\right)(-i)^{n} J_{n}\left(u_{0}\right) \cosh \left(n \frac{\hbar \beta \Omega_{1}}{2}\right)},
$$

$$
\begin{gathered}
\Delta_{n, s}=\Delta \exp (Y / 2) \sqrt{\left(2-\delta_{n, 0}\right)(-i)^{n} J_{n}\left(u_{0}\right) \sinh \left(n \frac{\hbar \beta \Omega_{1}}{2}\right)}, \\
Y=-W \frac{\sinh \left(\beta \hbar \Omega_{1}\right)}{\cosh \left(\beta \hbar \Omega_{1}\right)-1} \\
W=\frac{4 g^{2} n_{1}(0)^{4}}{\Omega_{1} \Omega\left[2 n_{\mathrm{th}}\left(\Omega_{1}\right)+1\right]}
\end{gathered}
$$

and

$u_{0}=i \sqrt{Y^{2}-W^{2}}=i\left(\frac{4 g^{2} n_{1}(0)^{4}}{\left[2 n_{\mathrm{th}}\left(\Omega_{1}\right)+1\right] \Omega_{1} \Omega}\right) \frac{1}{\sinh \left(\beta \hbar \Omega_{1} / 2\right)}$.

To obtain analytical expressions, we observe that, as discussed in Secs. III and IV, our expression for the effective spectral density is justified when $g / \Omega \ll 1$ and $\beta \hbar \Omega_{1}>1$. In this regime, the inequality $\left|u_{0}\right|<1$ holds. As suggested in [28], this allows effectively a truncation to the $n=0$ and $n=1$ contributions in $K^{(s)}(\lambda)$, because the argument of the Bessel functions is small, leading to the following approximations:

$$
\begin{gathered}
\Delta_{0, c}^{2}=\Delta^{2} \exp (Y) J_{0}\left(u_{0}\right) \approx \Delta^{2}\left(1-\frac{4 g^{2} n_{1}(0)^{6}}{\Omega^{2}}\right), \\
\Delta_{1, c}^{2}=\Delta^{2} \exp (Y) \sqrt{Y^{2}-W^{2}} \cosh \left(\beta \hbar \Omega_{1} / 2\right) \approx \Delta^{2} \frac{4 g^{2} n_{1}(0)^{6}}{\Omega^{2}},
\end{gathered}
$$

where corrections of order $O\left(g^{4} / \Omega^{4}\right)$ have been neglected. Notice also that, because our theory only accounts for corrections up to linear order in the nonlinearity $\alpha$, is $n_{1}(0)^{6}=1-$ $6(3 \alpha / 2 \hbar \Omega)+O\left(\alpha^{2}\right)$. Solving the undamped pole equation yields

$$
\begin{aligned}
\lambda_{p}^{2} \equiv & \lambda_{ \pm}^{2}=-\frac{\Delta_{0, c}^{2}+\Delta_{1, c}^{2}+\Omega_{1}^{2}}{2} \\
& \pm \sqrt{\left(\frac{\Delta_{0, c}^{2}-\Omega_{1}^{2}}{2}\right)^{2}+\frac{\Delta_{1, c}^{2}}{2}\left(\Delta_{0, c}^{2}+\frac{\Delta_{1, c}^{2}}{2}+\Omega_{1}^{2}\right)} \\
& :=-\Omega_{ \pm}^{2} .
\end{aligned}
$$


The last two equations allow the determination of the oscillation frequency. Finally, within the WDA, the qubit's population difference is obtained as

$$
\begin{aligned}
P(t)= & \exp \left(-\gamma \kappa_{-} t\right) \frac{\lambda_{-}^{2}+\Omega_{1}^{2}}{\lambda_{-}^{2}-\lambda_{+}^{2}}\left[\cos \left(\Omega_{-} t\right)-\frac{\gamma \kappa_{-}}{\Omega_{-}} \sin \left(\Omega_{-} t\right)\right] \\
& +\exp \left(-\gamma \kappa_{+} t\right) \frac{\lambda_{+}^{2}+\Omega_{1}^{2}}{\lambda_{+}^{2}-\lambda_{-}^{2}}\left[\cos \left(\Omega_{+} t\right)-\frac{\gamma \kappa_{+}}{\Omega_{+}} \sin \left(\Omega_{+} t\right)\right],
\end{aligned}
$$

where $\kappa_{ \pm}=\kappa\left(\lambda_{ \pm}\right)$, which is derived in detail in Eq. (B1) of Ref. [28].

We consider two possible resonance cases: First, we choose the resonance condition $\Omega_{1}=\Delta_{0, c}$, such that the oscillation frequencies are, to lowest order in $g / \Omega$ and $\alpha / \hbar \Omega$,

$$
\begin{aligned}
\Omega_{ \pm} & =\Omega_{1} \mp \frac{\Delta_{1, c}}{2} \\
& =\Omega+\frac{3}{\hbar} \alpha \mp g\left(1-\frac{3 \alpha}{2 \hbar \Omega}\right) .
\end{aligned}
$$

As a consequence, we obtain the so-called Bloch-Siegert shift:

$$
\Omega_{-}-\Omega_{+}=2 g(1-3 \alpha / 2 \hbar \Omega),
$$

which has the same form as in Ref. [31]. For comparison with [31], we also choose as second condition $\Delta=\Omega$, such that, to lowest order in $g / \Omega$ and $\alpha / \hbar \Omega$, we obtain

$$
\begin{gathered}
\Omega_{ \pm}=\Omega+\frac{3 \alpha}{2 \hbar} \mp g\left(1-\frac{9 \alpha g}{4 \hbar \Omega}\right), \\
\Omega_{-}-\Omega_{+}=2 g\left(1-\frac{9 \alpha g}{4 \hbar \Omega}\right),
\end{gathered}
$$

which also agrees with the results of [31].

We show in Figs. 4 and 5 a comparison of the analytic WDA formula Eq. (48), the numerical solution of the NIBA Eq. (34), denoted by NIBA, and the results obtained in Ref. [31] from a numerical solution of the Bloch-Redfield equations and referred to as the TLS-NLO approach. We observe that the dynamics are dominated by two frequencies and are well reproduced within all three approaches. In the

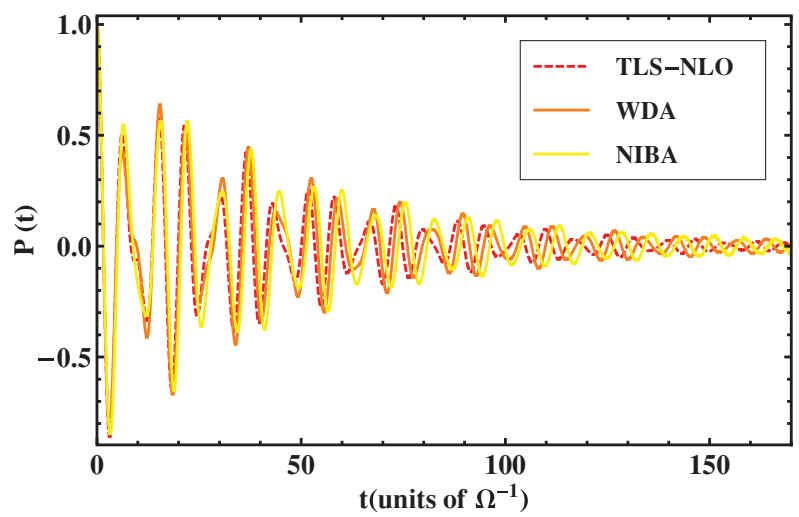

FIG. 4. (Color online) Comparison of the behavior of $P(t)$ as obtained from the numerical solution of the Bloch-Redfield equations based on the TLS-NLO approach [Ref. [31]], the numerical solution of the NIBA equation [Eq. (34)], and the analytical formula provided in Eq. (48). The chosen parameters are $\alpha=0.02(\hbar \Omega)$, $g=0.18 \Omega, \varepsilon=0, \Delta=\Omega, \gamma /(2 \pi \Omega)=0.0154$, and $\beta=10(\hbar \Omega)^{-1}$. The dynamics agree within all three approaches.

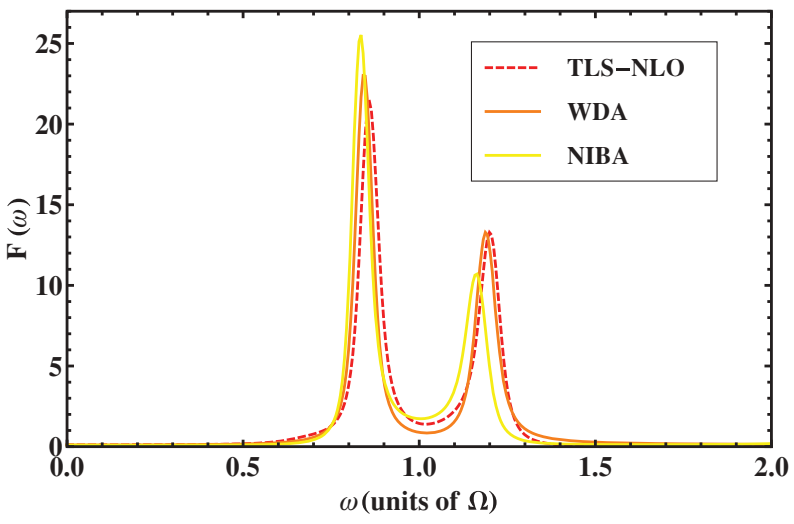

FIG. 5. (Color online) Corresponding Fourier transform of $P(t)$ as shown in Fig. 4.

Fourier spectrum, we observe tiny deviations of the resonance frequencies. They are due to the fact that the coupling strength $g$ is large enough that higher orders in the coupling yield a finite contribution in the effective-bath description. However, as we derived above when expanding the analytic formula Eq. (47), we find the same results up to lowest order in the coupling $g$ and in the nonlinearity $\alpha$. We emphasize that this small discrepancy is also seen for the corresponding linear system in the work of Hausinger et al. [37] when comparing the NIBA results in Ref. [28] with those of the Bloch-Redfield procedure. Finally, we also consider the regime where the coupling is much weaker than the nonlinearity $(\hbar g \ll \alpha)$. Then Eq. (32) of Ref. [31] has to be expanded differently; in this regime the results of Eq. (41) in Ref. [31] are not applicable. Rather, a proper expansion in this regime allows us to neglect $O\left(g^{2}\right)$, or higher if $O\left(\alpha^{2}\right)$ is neglected. The transition frequencies, when choosing $\Omega=\Delta$, follow from Eq. (32) of Ref. [31] and read

$$
\begin{aligned}
\Omega_{ \pm} & =\Omega+\frac{3}{2 \hbar} \alpha \mp \frac{1}{2} \sqrt{9 \alpha^{2} / \hbar^{2}} \\
& =\left\{\begin{array}{l}
\Omega, \\
\Omega_{1}=\Omega+3 \alpha / \hbar .
\end{array}\right.
\end{aligned}
$$

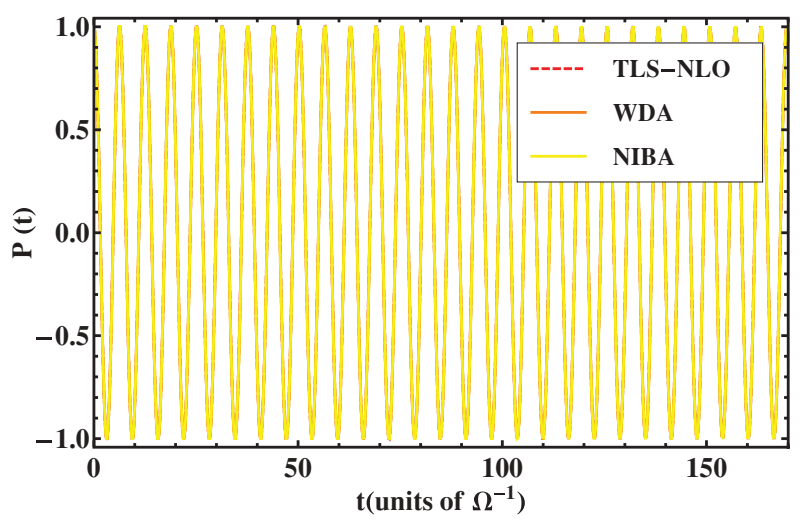

FIG. 6. (Color online) As in Fig. 4 but for the smaller TLS-NLO coupling constant $g=0.0018 \Omega$. 


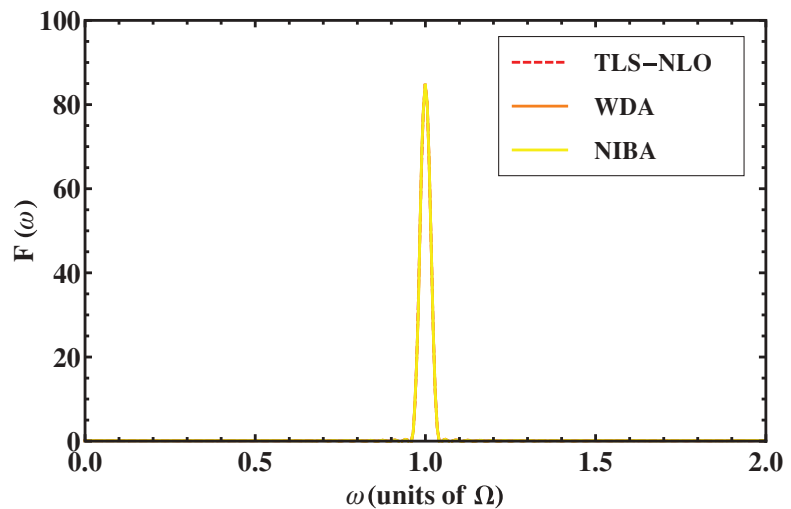

FIG. 7. (Color online) Corresponding Fourier transform of $P(t)$ shown in Fig. 6.

Alternatively, we can also perform an expansion of Eq. (47) consistent with this parameter regime and obtain

$$
-\Omega_{ \pm}^{2}=\frac{1}{2}\left(-\Omega^{2}-\Omega_{1}^{2} \pm \Omega^{2} \mp \Omega_{1}^{2}\right),
$$

such that

$$
\Omega_{+}=\Omega, \quad \Omega_{-}=\Omega_{1}=\Omega+3 \alpha / \hbar .
$$

The transition frequencies in Eqs. (53) and (56) coincide, and in Figs. 6 and 7 no deviation is observed when comparing the three different approaches.

\section{Influence on the qubit dynamics due to the nonlinearity-a comparison of the NIBA for linear and nonlinear effective spectral densities}

In this last section we wish to address the effects of the nonlinearity on the qubit dynamics. The comparison of linear versus nonlinear case is done at the level of the numerical solution of the NIBA equation and shown in Figs. 8 and 9. As already obtained in Ref. [31], we observe that the transition frequencies are shifted to higher values compared to the linear case. Specifically, as can be seen from Eqs. (49) and (52), the reduction is linear in $\alpha$. Also as a consequence, the amplitudes associated with the transitions are modified. Moreover, we observe a decrease of the vacuum Rabi splitting compared to

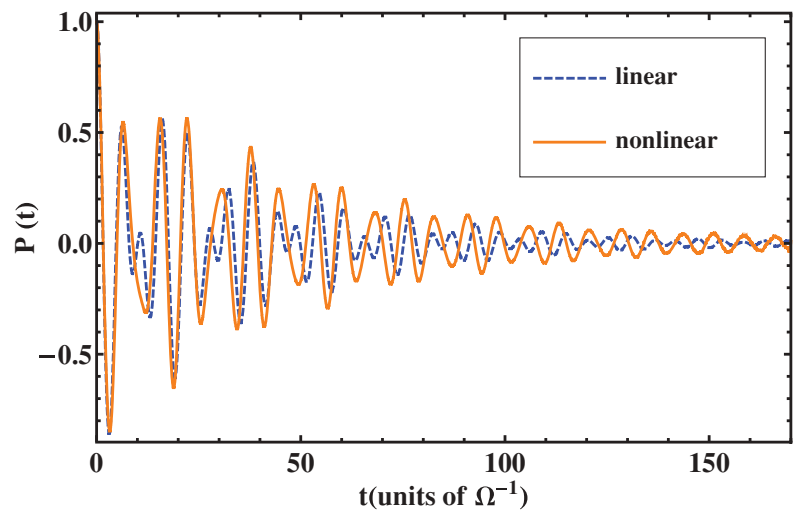

FIG. 8. (Color online) $P(t)$ within the NIBA when using the linear and the nonlinear effective spectral densities, Eqs. (13) and (28), respectively. Parameters are $\alpha=0.02(\hbar \Omega)$ or $\alpha=0$ respectively, $g=$ $0.18 \Omega, \varepsilon=0, \gamma /(2 \pi \Omega)=0.0154$, and $\beta=10(\hbar \Omega)^{-1}$.

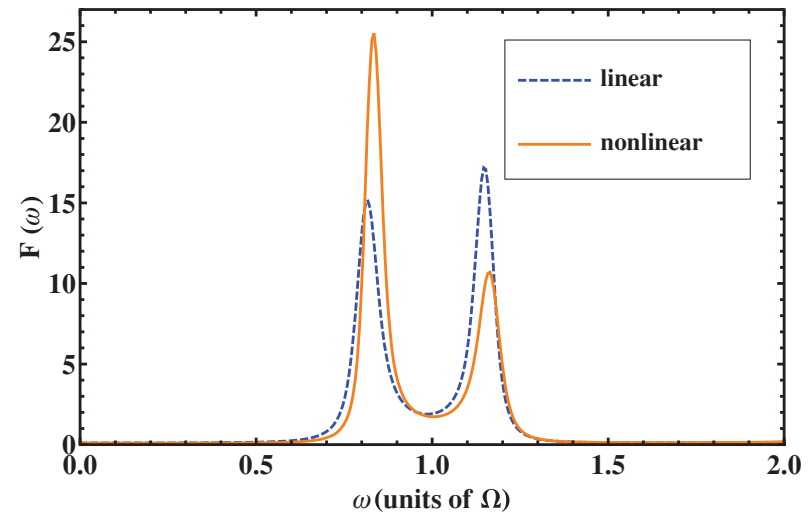

FIG. 9. (Color online) Corresponding Fourier transform of $P(t)$ shown in Fig. 8. The effect of the nonlinearity is to increase the resonance frequencies with respect to the linear case. As a consequence the relative peak heights change.

the linear case; see Eqs. (50) and (52). Consequently, the effect of the nonlinearity of the read-out device can be observed in the qubit dynamics.

\section{CONCLUSIONS}

In this work we determined the dynamics of a qubit coupled via a nonlinear oscillator (NLO) to an Ohmic bath within an effective-bath description. We investigated an approximate mapping procedure based on linear response theory, which is applicable for the case of weak nonlinearities $\alpha$ and small to moderate qubit-NLO coupling $g$. We determined the effective spectral density in terms of the qubit-oscillator coupling and the linear susceptibility of a nonlinear oscillator. The susceptibility was calculated for practical purposes from the periodically driven counterpart of the original nonlinear oscillator, yielding an analytical expression for the effective spectral density valid at low temperatures. The spectral density thus obtained shows resonant behavior. In particular, it has almost a Lorentzian form for the parameter regime considered and is peaked at a shifted frequency; namely, at the one-photon resonance between the ground state and first-excited state of the nonlinear oscillator. Moreover, this effective spectral density acquires a temperature dependence and is Ohmic at low frequencies. Based on the effective spectral density, the qubit dynamics are investigated within the NIBA approximation. In addition, an analytical formula for the qubit dynamics is provided, which describes very well the dynamics at low damping.

These results were compared to numerical predictions from Ref. [31], where the Bloch-Redfield equations for the density matrix of the coupled qubit nonlinear oscillator system (TLSNLO) are solved. These latter equations have the same regime of validity as those of the effective-bath approach; namely, weak nonlinearities $\alpha / \hbar \Omega \ll 1$, small qubit-nonlinear oscillator coupling $g / \Omega \ll 1$, and low temperatures $k_{B} T / \hbar \Omega \ll 1$. Thus, in this parameter regime, an overall agreement of the two approaches is expected. Exemplarily, the predictions of the two approaches were analyzed for two possible coupling strengths $g$. We emphasize that parameters like temperature and damping and especially the strength of the coupling $g$ 
and nonlinearity $\alpha$ determine the appropriate form of the expansions in the different parameter regimes. Due to the tunability of the parameters, various qubit dynamics are possible. Near and at resonance, the same analytical results are predicted within the two approaches up to first order in the coupling and nonlinearity. In agreement with Ref. [31], we observed the following effects due to the nonlinearity: In the regime $g \gg \alpha / \hbar$, a Rabi vacuum splitting is observed. The transition frequencies of the two dominating peaks are shifted to larger values compared to the linear case. Also as a consequence, the amplitudes of the coherent oscillations of the population difference $P(t)$ are modified. Moreover, the Bloch-Siegert shift is decreased due to the nonlinearity. In the regime $g \ll \alpha / \hbar$, only one peak is predicted.

We conclude that, as in case of the corresponding linear system [28,37], the effective-bath description provides an alternative approach to investigate the complex dynamics of the qubit-dissipative NLO system.

\section{ACKNOWLEDGMENT}

We acknowledge support by the DFG under the funding program SFB 631.

\section{APPENDIX: BATH CORRELATION FUNCTIONS}

We now consider the qubit dynamics for the case of the effective nonlinear bath. Therefore, we determine the actual form of the correlation functions $S(\tau)$ and $R(\tau)$. From Eq. (37) it follows

$$
\begin{gathered}
S(\tau)=X \tau+L\left[\exp \left(-\bar{\gamma}_{\mathrm{th}} \tau\right) \cos \left(\Omega_{1} \tau\right)-1\right] \\
+Z \exp \left(-\bar{\gamma}_{\mathrm{th}} \tau\right) \sin \left(\Omega_{1} \tau\right), \\
R(\tau)=I-\exp \left(-\bar{\gamma}_{\mathrm{th}} \tau\right)\left[N \sin \left(\Omega_{1} \tau\right)\right. \\
\left.\quad+I \cos \left(\Omega_{1} \tau\right)\right],
\end{gathered}
$$

where

$$
\begin{gathered}
I=\frac{2 \pi \varsigma \Omega^{2}}{\Omega_{1}^{2}+\bar{\gamma}_{\mathrm{th}}^{2}}, \\
N=-I\left(\frac{\Omega_{1}}{\bar{\gamma}_{\mathrm{th}}}-\frac{\bar{\gamma}_{\mathrm{th}}}{\Omega_{1}}\right), \\
X=\frac{2}{\hbar \beta} I,
\end{gathered}
$$

$$
\begin{aligned}
L= & -\left(\frac{I}{\bar{\gamma}_{\mathrm{th}}}\right) \frac{1}{\cosh \left(\beta \hbar \Omega_{1}\right)-\cos \left(\beta \hbar \bar{\gamma}_{\mathrm{th}}\right)} \\
& \times\left[\Omega_{1} \sinh \left(\beta \hbar \Omega_{1}\right)-\bar{\gamma}_{\mathrm{th}} \sin \left(\beta \hbar \bar{\gamma}_{\mathrm{th}}\right)\right], \\
Z= & -\left(\frac{I}{\bar{\gamma}_{\mathrm{th}}}\right) \frac{1}{\cosh \left(\beta \hbar \Omega_{1}\right)-\cos \left(\beta \hbar \bar{\gamma}_{\mathrm{th}}\right)} \\
& \times\left[\bar{\gamma}_{\mathrm{th}} \sinh \left(\beta \hbar \Omega_{1}\right)+\Omega_{1} \sin \left(\beta \hbar \bar{\gamma}_{\mathrm{th}}\right)\right] .
\end{aligned}
$$

Here, we have neglected the contribution coming from the Matsubara term, which is verified if the temperature is high enough [2] [i.e., $k_{B} T \gg \hbar \bar{\gamma} /(2 \pi)$ ]. Moreover, we applied in the contributions of the poles lying in the vicinity of $\pm \Omega_{1}$ the approximation: $2 \Omega_{1} /\left(2 \Omega_{1} \pm i \bar{\gamma}_{\text {th }}\right) \approx 1$. This corresponds effectively to the neglect of certain $O\left(\bar{\gamma}_{\text {th }}\right)$ contributions.

The above expressions can be linearized in the damping:

$$
\begin{aligned}
& S(\tau)=S_{0}(\tau)+S_{1}(\tau)+O\left(\gamma^{2}\right), \\
& R(\tau)=R_{0}(\tau)+R_{1}(\tau)+O\left(\gamma^{2}\right),
\end{aligned}
$$

with

$$
\begin{gathered}
S_{0}(\tau)=Y\left[\cos \left(\Omega_{1} \tau\right)-1\right], \\
S_{1}(\tau)=A \tau \cos \left(\Omega_{1} \tau\right)+B \tau+C \sin \left(\Omega_{1} \tau\right), \\
R_{0}(\tau)=W \sin \left(\Omega_{1} \tau\right), \\
R_{1}(\tau)=V\left[1-\cos \left(\Omega_{1} \tau\right)-\frac{\Omega_{1} \tau}{2} \sin \left(\Omega_{1} \tau\right)\right] .
\end{gathered}
$$

The zeroth-order coefficients in the damping are given by

$$
\begin{aligned}
& Y=-W \frac{\sinh \left(\beta \hbar \Omega_{1}\right)}{\cosh \left(\beta \hbar \Omega_{1}\right)-1}, \\
& W=\frac{4 g^{2} n_{1}(0)^{4}}{\Omega_{1} \Omega\left[2 n_{\mathrm{th}}\left(\Omega_{1}\right)+1\right]},
\end{aligned}
$$

and the first-order coefficients are given by

$$
\begin{gathered}
A=-\bar{\gamma}_{\mathrm{th}} Y, \\
B=\frac{2}{\hbar \beta} V, \\
C=-V \frac{\beta \hbar \Omega_{1}+\sinh \left(\beta \hbar \Omega_{1}\right)}{\left[\cosh \left(\beta \hbar \Omega_{1}\right)-1\right]\left[2 n_{\mathrm{th}}\left(\Omega_{1}\right)+1\right]}, \\
V=\frac{2 g^{2} n_{1}(0)^{4} \gamma}{\Omega_{1}^{2} \Omega} .
\end{gathered}
$$

[1] M. A. Nielson and I. L. Chuang, Quantum Computation and Quantum Information (Cambridge University Press, 2000).

[2] U. Weiss, Quantum Dissipative Systems, 3rd ed. (World Scientific, Singapore, 2008).

[3] A. J. Leggett, S. Chakravarty, A. T. Dorsey, M. P. A. Fisher, A. Garg, and W. Zwerger, Rev. Mod. Phys. 67, 725 (1995).

[4] M. Grifoni and P. Hänggi, Phys. Rep. 304, 229 (1998).

[5] A. G. Redfield, IBM J. Res. Dev. 1, 19 (1957).

[6] K. Blum, Density Matrix Theory and Applications, 2nd ed. (Plenum Press, New York, London, 1996).

[7] L. Hartmann, I. Goychuk, M. Grifoni, and P. Hänggi, Phys. Rev. E 61, R4687 (2000).
[8] J. E. Mooij, T. P. Orlando, L. Levitov, L. Tian, C. H. van der Wal, and S. Lloyd, Science 285, 1036 (1999).

[9] L. Tian, S. Lloyd, and T. P. Orlando, Phys. Rev. B 65, 144516 (2002).

[10] C. van der Wal, F. Wilhelm, C. J. P. M. Harmans, and J. E. Mooij, Eur. Phys. J. B 31, 111 (2003).

[11] I. Chiorescu, Y. Nakamura, J. P. M. Harmans, and J. E. Mooij, Science 299, 1869 (2003).

[12] I. Chiorescu, P. Bertet, K. Semba, Y. Nakamura, J. P. M. Harmans, and J. E. Mooij, Nature (London) 431, 159 (2004).

[13] J. Johansson, S. Saito, T. Meno, H. Nakano, M. Ueda, K. Semba, and H. Takayanagi, Phys. Rev. Lett. 96, 127006 (2006). 
[14] A. Lupaşcu, J. P. M. Harmans, and J. E. Mooij, Phys. Rev. B 71, 184506 (2005).

[15] J. C. Lee, W. D. Oliver, K. K. Berggren, and T. P. Orlando, Phys. Rev. B 75, 144505 (2007).

[16] T. Picot, A. Lupaşcu, S. Saito, J. P. M. Harmans, and J. E. Mooij, Phys. Rev. B 78, 132508 (2008).

[17] A. Lupaşcu, E. F. C. Driessen, L. Roschier, C. J. P. M. Harmans, and J. E. Mooij, Phys. Rev. Lett. 96, 127003 (2006).

[18] K. Inomata, M. Watanabe, T. Yamamoto, K. Matsuba, Y. Nakamura, and J. S. Tsai, J. Phys. Conference Series 150, 052077 (2009).

[19] T. Wirth, J. Lisenfeld, A. Lukashenko, and A. V. Ustinov, e-print arXiv: $1010.0954 \mathrm{v} 1$.

[20] I. Siddiqi, R. Vijay, F. Pierre, C. M. Wilson, L. Frunzio, M. Metcalfe, C. Rigetti, R. J. Schoelkopf, M. H. Devoret, D. Vion, and D. Esteve, Phys. Rev. Lett. 94, 027005 (2005).

[21] I. Siddiqi, R. Vijay, M. Metcalfe, E. Boaknin, L. Frunzio, R. J. Schoelkopf, and M. H. Devoret, Phys. Rev. B 73, 054510 (2006).

[22] F. Mallet, F. R. Ong, A. Palacios-Laloy, F. Nguyen, P. Bertet, D. Vion, and D. Esteve, Nat. Phys. 5, 791 (2009).

[23] A. Garg, J. N. Onuchic, and V. Ambegaokar, J. Chem. Phys. 83, 4491 (1985).

[24] F. K. Wilhelm, S. Kleff, and J. von Delft, Chem. Phys. 296, 345 (2004).
[25] M. C. Goorden, M. Thorwart, and M. Grifoni, Eur. Phys. J. B 45, 405 (2005).

[26] M. C. Goorden, M. Thorwart, and M. Grifoni, Phys. Rev. Lett. 93, 267005 (2004).

[27] M. C. Goorden and F. K. Wilhelm, Phys. Rev. B 68, 012508 (2003).

[28] F. Nesi, M. Grifoni, and E. Paladino, New J. Phys. 9, 316 (2007).

[29] S. Kleff, S. Kehrein, and J. von Delft, Phys. Rev. B 70, 014516 (2004).

[30] F. Brito and A. O. Caldeira, New J. Phys. 10, 115014 (2008)

[31] C. Vierheilig, J. Hausinger, and M. Grifoni, Phys. Rev. A 80, 052331 (2009).

[32] V. Volterra, Theory of Functionals and of Integral and IntegroDifferential Equations (Dover Publications, New York, 1959).

[33] V. Peano and M. Thorwart, New J. Phys. 8, 21 (2006).

[34] C. Vierheilig and M. Grifoni, Chem. Phys. 375, 216 (2010).

[35] A. Wallraff, D. I. Schuster, A. Blais, J. M. Gambetta, J. Schreier, L. Frunzio, M. H. Devoret, S. M. Girvin, and R. J. Schoelkopf, Phys. Rev. Lett. 99, 050501 (2007).

[36] M. Grifoni, M. Sassetti, and U. Weiss, Phys. Rev. E 53, R2033 (1996).

[37] J. Hausinger and M. Grifoni, New J. Phys. 10, 115015 (2008). 